\title{
Analysis of Kinematic Data in Pathological Tremor with the Hilbert-Huang Transform
}

\author{
J.A. Gallego, Student Member, IEEE, E. Rocon, Member, IEEE, A.D. Koutsou, and J.L. Pons, Member, IEEE
}

\begin{abstract}
This paper presents analysis of kinematic data of tremor patients while performing different tasks with Ensemble Empirical Mode Decomposition (EEMD), a novel noise-assisted data analysis method. EEMD automatically separates raw kinematic data into three components: 1) noise from various sources, 2) tremulous movement, and 3) voluntary movement. Comparison of this technique with other decomposition methods such as recursive forth and back filters or Empirical Mode Decomposition (EMD) shows a better performance; EEMD separation of tremor diminishes EMD error in a $45.2 \%$ (mean error $0.041 \pm 0.036 \mathrm{rad} / \mathrm{s}$ ). Moreover, postprocessing of EEMD separated tremor allows the calculation of the Hilbert spectrum, a high resolution time-energy-frequency distribution that improves analysis of tremors.
\end{abstract}

\section{INTRODUCTION}

Tremor, defined as a rhythmic oscillatory activity of body parts [1], constitutes the most extended movement disorder. Although tremor is not life threatening, it is source of both functional disability and social embarrassment. It is accepted that tremor is generated by different combinations of four physiologic mechanisms: 1) oscillations due to central generators, 2) oscillations because of distorted feedback or feedforward loops, 3) oscillations based on reflexes, and 4) mechanical oscillations, [1]. Nevertheless, the specific pathophysiology of the different disorders that cause tremor is yet far from completely understood, making misdiagnosis exceedingly common, [2]. This fact encourages research in novel analysis and modeling techniques.

From a data analysis perspective, tremor time series are typically studied employing methods based on Fourier analysis. This approach has many drawbacks related to the linearity and stationarity hypotheses inherent to Fourier spectral analysis, because tremors constitute time varying phenomena [3], which can be formulated as nonlinear stochastic processes, [4]. Traditional assessment with amplitude spectra may yield confusing results because, for example, it is hard to interpret whether different peaks represent coexistence of separate tremor mechanisms, or appear due to frequency fluctuations, [5]. Alternate approaches such as spectrograms built upon overlapping Short Time Fourier Transforms, or the Weighted Frequency Fourier Linear Combiner [6], yield

The work presented in this paper has been carried out with the financial support from the Commission of the European Union, within Framework 7, specific IST programme "Accessible and Inclusive ICT", Target outcome 7.2 "Advanced self-adaptive ICT-enabled assistive systems based on noninvasive Brain to Computer Interaction (BCI)", under Grant Agreement number ICT-2007-224051, "TREMOR: An ambulatory BCI-driven tremor suppression system based on functional electrical stimulation."

The authors are with the Bioengineering Group, Consejo Superior de Investigaciones Científicas, CSIC, Arganda del Rey, Madrid, 28500 Spain, e-mail: gallego@iai.csic.es. more reliable results; the former provides a time-amplitudefrequency representation of the signal, whereas the latter tracks instantaneous tremor amplitude and frequency adapting to their variations, but assuming that tremor is due to an unique oscillator.

In a recent work, [3], we have proposed the use of Empirical Mode Decomposition (EMD) as a novel technique for the study of kinematic tremor data. EMD is a data driven sifting (decomposition) technique that makes no a priori assumptions on the input signals [7], thus it is suitable for the analysis of nonlinear and non-stationary processes. The output of EMD is a number of intrinsic mode functions (IMFs) that admit well-behaved Hilbert transform, allowing for precise representation in the time-energy-frequency domain, the so called Hilbert spectrum. In [3], it was demonstrated that EMD automatically separates joint rotation into concomitant voluntary and tremulous components with very small discrepancy when compared to manual decomposition with recursive forth and back filters.

This article presents the application of Ensemble Empirical Mode Decomposition (EEMD), a new improvement to the original EMD algorithm that relies on sifting an ensemble of white noise-added signal [8]. This approach overcomes mode mixing, understood as: 1) having IMFs that consist of signals of widely disparate scales, or 2) a signal of a similar scale residing in different IMF components. The latter phenomenon is of pivotal importance when analyzing some tasks carried out by tremor patients, because wrist motion during activities of daily living (ADL) may have a part of their energy around $4-5 \mathrm{~Hz}$, close to tremor frequency, which typically lies between 3 and $12 \mathrm{~Hz}$ [1] (although the predominant peak of voluntary movements during ADL is between 0.48 and $2.47 \mathrm{~Hz}$ [9]).

Hence, here we present decomposition of tremor time series with EEMD, and compare the results obtained with those provided by EMD. Our results indicate that EEMD outperforms EMD by decreasing the Filtered Mean Square Error with Delay correction, $\mathrm{FMSE}_{\mathrm{d}}$, a metric for quantification of tremor estimates [10] by a $45.2 \%$, yielding a mean error of $0.041 \pm 0.036 \mathrm{rad} / \mathrm{s}$. We also found a discrepancy with the results in [3]: tremor decomposition with EEMD typically indicates that tremor appears in more than one component, which may be related to the presence of different oscillators in the tremor genesis.

\section{MATERiAls}

This paper presents data from four patients, two of them suffering from Essential tremor (ET), one from Parkinson's 

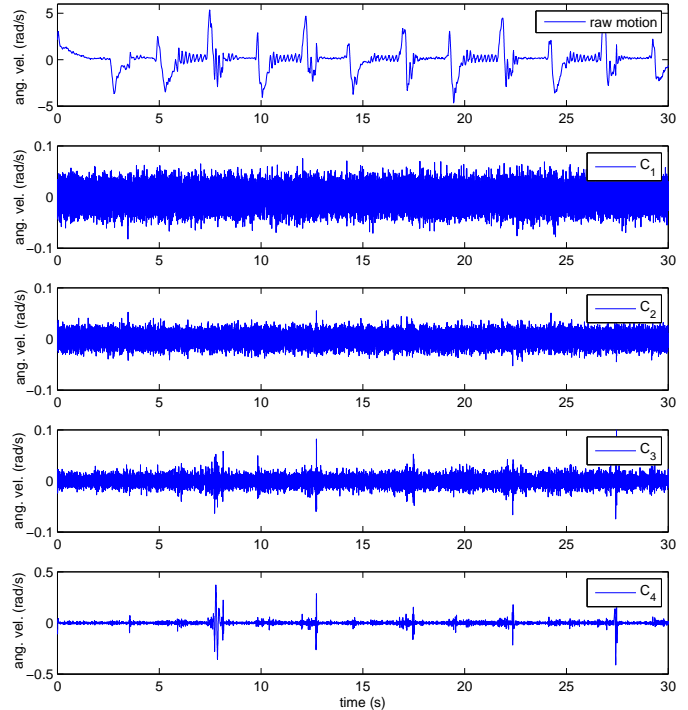

(a)
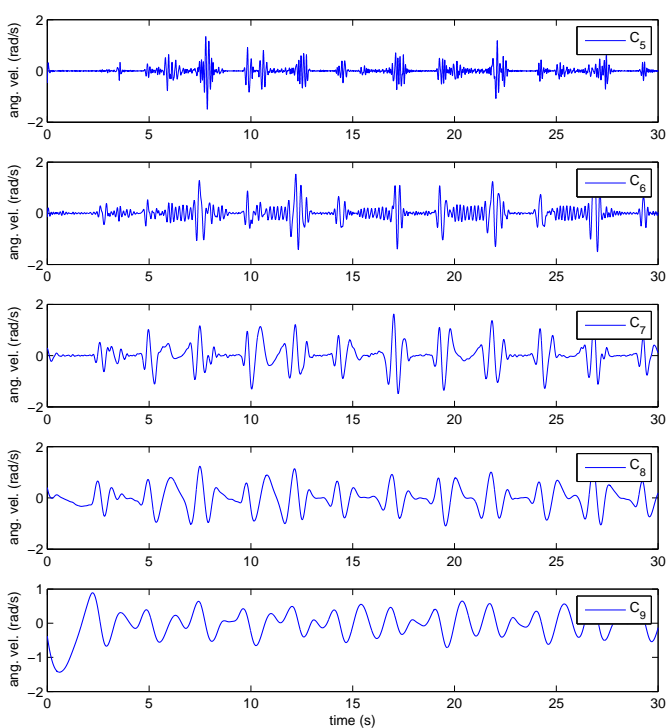

(b)

Fig. 1. Wrist rotation recorded during continuous execution of the finger to nose test by patient A. Fig. (a) shows the original signal together with the first four components provided by EEMD, whereas (b) shows components five to nine, both included.

disease (PD), and one from Extrapyramidal Syndrome (ES). Both ET patients (patients A and B) had unilateral postural and kinetic tremor of mild severity, i.e. grade 2 according to Faher scale. The PD patient (patient $\mathrm{C}$ ) had bilateral rest and postural tremor of grades 3 and 1 respectively. Finally, the ES patient (patient D) had bilateral rest and postural tremor of grades 2 and 1 respectively. Medications were continued at the time of the recordings. The Ethical Committee at Hôptial Erasme gave ethical approval for this study.

During the measurements, patients were comfortably seated on a chair. Each patient performed three repetitions of four tasks; three of them selected because they activate different types of tremor, while the last one was included regarding functional analysis. The tasks were: 1) holding both arms against gravity (AG), 2) resting the arm on the lap (RE), 3) touching the nose and knee alternatively with the fingertip (NK), and 4) pouring water from a bottle into a glass (WG). Average task duration was $\sim 30 \mathrm{~s}$.

Wrist tremor was assessed with inertial sensors. We employed differential measurement of hand and forearm rotation to obtain wrist flexion extension, as described in previous works, [11], [3]. Inertial sensors employed (TechMCS, Technaid S.L., Madrid, Spain) have small size (27 x 35 x $13 \mathrm{~mm}$ ) and low weight $(27 \mathrm{~g})$, fundamental requirements for this kind of application.

\section{DATA ANALYSIS}

EMD is an iterative technique that sifts an input signal into a finite number of IMFs, a series of functions that satisfy two conditions derived from the notion of instantaneous frequency as defined in [7]: 1) in the whole data set the number of extrema and zero crossings must be the same or differ by one, and 2) at every point, the mean value of the envelop defined by the local maxima and the local minima must be zero. As the so called sifting process is iterative, once the first IMF is extracted, the residual is sifted to generate the second IMF. This procedure continues until a certain stop criterion is fulfilled, typically defined as the size of the standard deviation of two consecutive sifting results [7], [3].

EEMD extends EMD by incorporating the concept of noise-assisted data analysis, understood as adding white noise with a certain covariance to the input signal. The effect of this added white noise is to provide a uniform reference frame in the time-frequency space [8]. This operation obviously yields noisier results, but the ensemble mean obtained after repeating it a number of times (typically a few hundred times) cancels out the added noise, thus providing the true answer. The improvement with respect to EMD is that by adding finite noise, EEMD eliminates mode mixing while preserving the uniqueness of decomposition. Note that the components provided by EEMD are not necessarily IMFs, as they are obtained as the ensemble mean of a number of IMFs. Therefore, to analyze the resulting components with the Hilbert transform, postprocessing with EMD is required [8].

EEMD has two parameters that need to be selected, the number of ensemble, $N$, and the noise amplitude, $\epsilon$. The former indicates how many siftings of white noise added data are executed before obtaining the ensemble mean, whereas the latter defines the covariance of the added noise. Both parameters provide the final standard deviation of error, $\epsilon_{n}$ :

$$
\epsilon_{n}=N^{-\frac{1}{2}} \epsilon
$$



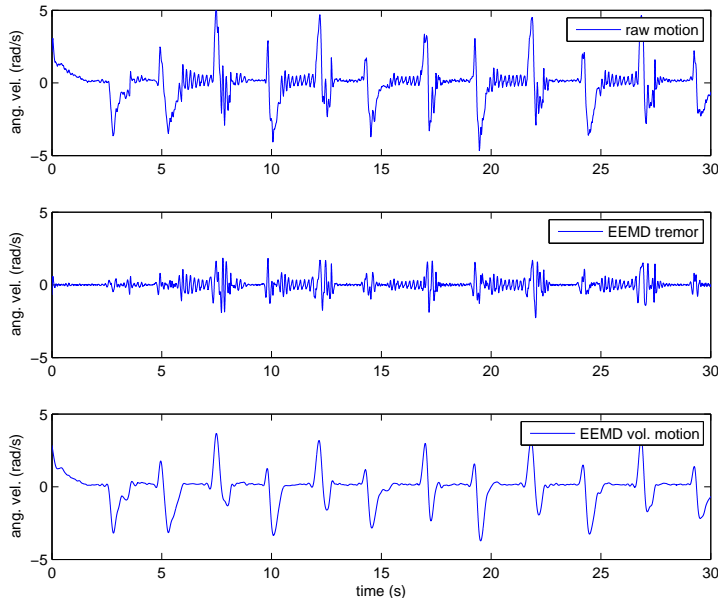

Fig. 2. EEMD decomposition of raw motion (top plot) in concomitant tremorous (middle plot) and voluntary movements (bottom plot), during a finger to nose test performed by patient A. Noise originated from the technique itself, sensor noise and skin fixation is removed by not considering the first three sifted components.

This work evaluates EEMD for automatic extraction of tremor sources embedded in joint rotations during execution of a certain task, and compares it with EMD. To allow for a reliable comparison, in both techniques the same Cauchy type stoppage criterion is used [7], [8]. Assessment is based on the $\mathrm{FMSE}_{\mathrm{d}}$ [10], a figure of merit specifically designed to assess tremor filters. The $\mathrm{FMSE}_{\mathrm{d}}$ consists in first aligning the estimated tremor with the reference signal, and afterwards computing the delay corrected estimation error (2). Reference tremor is obtained by executing a non causal recursive forth and back filter as in [3].

$$
\mathrm{FMSE}_{\mathrm{d}}=\mathrm{E}\left[s_{k}-t_{k-\hat{d}_{k}}\right]^{2}
$$

Where $s_{k}$ represents the reference tremor signal to be estimated, and $t_{k-\hat{d}_{k}}$ stands for the delay compensated tremor estimation. Instantaneous delay $\hat{d}_{k}$ is calculated by means of an adaptive algorithm that minimizes the mean square error function based on a LMS like recursion.

In this study, we analyse both with EMD and EEMD data of all four patients executing the tasks described above. EEMD parameters are set to: $N=100, \epsilon=0.2$, which yields very small standard deviation of error, $\epsilon_{n}=0.02$.

\section{RESUlTS}

Fig. 1 shows decomposition of wrist motion with EEMD during continuous execution of the finger to nose test by patient A. We observe that the three first components are related to noise originated from: 1) the white noise added because of the noise assisted nature of the technique, and 2) intrinsic gyroscope noise (which is also modeled as white noise, [12]). We hypothesize that component three also captures artifacts due to skin fixation, as evident peaks appear only at certain moments during execution of voluntary movement. Addition
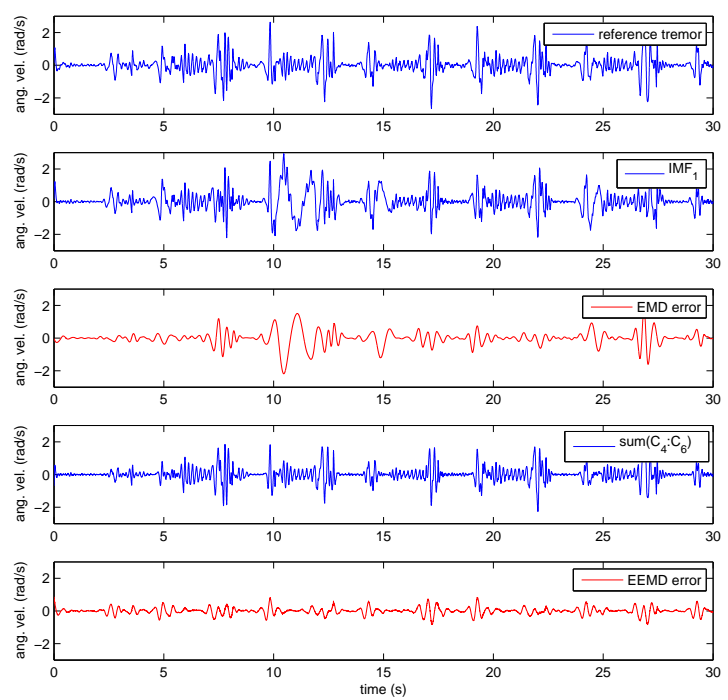

Fig. 3. Comparison of EMD and EEMD sifting of the signal shown in Fig. 1. The top plot shows reference tremor, whereas the middle and bottom panels depict EMD and EEMD sifting (blue) and their correspondent errors (red).

TABLE I

PERFORMANCE OF EMD AND EEMD FOR AUTOMATIC DECOMPOSITION OF TREMOR KINEMATIC DATA IN TERMS OF AVERAGE FMSE d $_{\mathrm{d}}(\mathrm{RAD} / \mathrm{S})$.

\begin{tabular}{clcccc}
\hline \multirow{2}{*}{ Patient } & \multirow{2}{*}{ Technique } & \multicolumn{4}{c}{ Task } \\
& & AG & NK & RE & WG \\
\hline \multirow{2}{*}{$\mathrm{A}$} & EMD & 0.038 & 0.187 & 0.021 & 0.109 \\
& EEMD & 0.022 & 0.139 & 0.009 & 0.064 \\
\hline \multirow{2}{*}{$\mathrm{B}$} & EMD & 0.060 & 0.094 & 0.021 & 0.113 \\
& EEMD & 0.030 & 0.072 & 0.013 & 0.057 \\
\hline \multirow{2}{*}{$\mathrm{C}$} & EMD & 0.011 & 0.045 & 0.028 & 0.113 \\
& EEMD & 0.007 & 0.034 & 0.016 & 0.057 \\
\hline \multirow{2}{*}{$\mathrm{D}$} & EMD & 0.024 & 0.190 & 0.090 & 0.056 \\
& EEMD & 0.014 & 0.082 & 0.015 & 0.027 \\
\hline
\end{tabular}

of components four to six represents tremor, whereas the sum of all the remaining ones (components seven to fifteen, some of them not shown here) yields concomitant voluntary motion, Fig. 2. Noise introduced by the technique itself and by the measurement setup is filtered out automatically by EEMD, because it corresponds to the first three components.

Comparison of EMD and EEMD for the same example, indicates that sifting of the signal with EEMD outperforms EMD, Fig. 3. In this case, EEMD provides a $\mathrm{FMSE}_{\mathrm{d}}=$ $0.132 \mathrm{rad} / \mathrm{s}$, versus $0.222 \mathrm{rad} / \mathrm{s}$ for EMD. Table I summarizes the $\mathrm{FMSE}_{\mathrm{d}}$ per patient and task for both techniques. We observe that EEMD performs better than EMD as it diminishes the $\mathrm{FMSE}_{\mathrm{d}}$ by a $45.2 \%$, yielding a mean error of $0.041 \pm$ $0.036 \mathrm{rad} / \mathrm{s}$.

\section{Discussion}

Previous sections presented analysis of tremor kinematic data with EEMD, a recent extension of EMD that consists in sifting an ensemble of noise added signal. The major advantage of this approach is that it avoids mode mixing, 


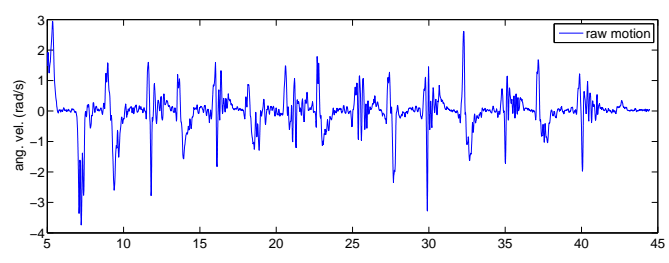

(a)

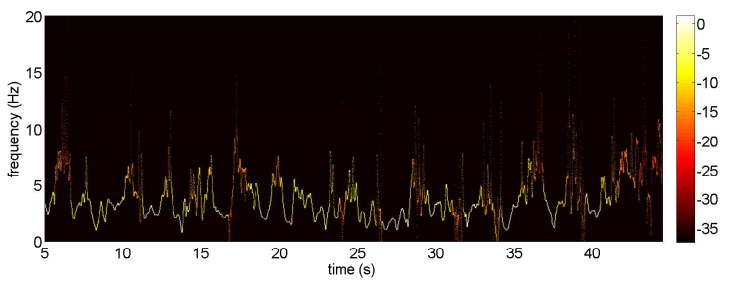

(b)

Fig. 4. Hilbert Spectrum (b) of the postprocessed EEMD tremor estimation during continuous execution of the finger to nose test by patient $\mathrm{B}$ (a).

which is of pivotal importance when analyzing relatively fast tasks carried out by tremor patients. As a matter of fact, mode mixing makes EMD identify fast voluntary movements as a constituent of moderate frequency tremors, degrading the performance of the method. A task that exemplifies this in our protocol is the finger to nose test (NK): Fig. 3 shows that EMD includes a relatively fast voluntary movement in the intrinsic mode function that describes tremor, $\mathrm{IMF}_{1}$ (time $\sim 10 \mathrm{~s}$ ), while EEMD ignores it and thus yields a better decomposition of movement. Similar phenomena are observed in all repetitions of this task, and also during the pouring water into a glass test (WG); Table I shows significant reduction in the $\mathrm{FMSE}_{\mathrm{d}}$ for both of them. General improvement of EEMD based separation with respect to EMD is also due to the intrinsic ability of the former to handle data containing white noise, because the ensemble mean not only removes the added noise, but also sensor noise and small vibrations due to skin fixation. These constituents are included in the first three EEMD components, Fig. 1.

Data analysis yields that, on the contrary to EMD, EEMD estimation of tremor is contained not in one, but in three components. We hypothesize that this may be related to different oscillators underlying tremor generation [13]. We plan future research on this topic.

As mentioned in Section III, analysis of EEMD separated tremor with the Hilbert transform requires previous sifting with EMD to generate IMFs. We have thus postprocessed the sum of the three components that cause tremor to obtain the Hilbert Spectrum (HS). Fig. 4 shows the HS during a finger to nose test carried out by patient B. Fluctuations in tremor frequency are well captured by the postprocessed EEMD tremor estimation. We observe that high frequency intervals coincide with movements (when kinetic tremor appears), whereas low frequency intervals are related to the maintenance of a posture, i.e. when the finger is touching the nose tip. This agrees with the neurological examination that reports $3 \mathrm{~Hz}$ postural tremor and $7 \mathrm{~Hz}$ kinetic tremor. The example illustrates the potential of analyzing EEMD tremor with the Hilbert transform.

\section{CONCLUSIONS}

This paper presented the analysis of tremor kinematic data with EEMD, a recent improvement to EMD, and compared their performance when separating tremor from concomitant voluntary movement. Evaluation with data from four tremor patients shows that EEMD outperforms EMD by reducing the $\mathrm{FMSE}_{\mathrm{d}}$, a figure of merit to assess the performance of tremor estimation algorithms, by a $45.2 \%$, yielding an average error of $0.041 \pm 0.036 \mathrm{rad} / \mathrm{s}$. We believe that the cause of this is twofold: first, the intrinsic noise-assisted nature of EEMD makes it remove noise from the original signal, therefore eliminating it from the tremor estimation; second, EEMD avoids mode mixing, which is troublesome in tasks that involve relatively fast voluntary movements, as the finger to nose test. Postprocessing of EEMD tremor components allows computation of the HS, a time-energyfrequency representation of the signal that provides better resolution than traditional analysis based on spectrograms. In our opinion, this technique may help to understand better the components of tremors.

\section{REFERENCES}

[1] G. Deuschl, J. Raethjen, M. Lindemann, and P. Krack, "The pathophysiology of tremor," Muscle \& Nerve, vol. 24, pp. 716-735, 2001.

[2] E. D. Louis, "Essential tremors: a family of neurodegenerative disorders?" Arch Neurol, vol. 66, no. 10, pp. 1202-1208, Oct 2009. [Online]. Available: http://dx.doi.org/10.1001/archneurol.2009.217

[3] E. Rocon, A. O. Andrade, J. L. Pons, P. Kyberd, and S. J. Nasuto, "Empirical mode decomposition: a novel technique for the study of tremor time series," Med Biol Eng Comput, vol. 44, pp. 569-582, 2006.

[4] J. Timmer, S. Häussler, M. Lauk, and C. H. Lücking, "Pathologic tremors: Deterministic chaos or nonlinear stochastic oscillators?" Chaos, pp. 278-288, 2000.

[5] M. Gresty and D. Buckwell, "Spectral analysis of tremor: Understanding the results," Journal of Neurology, Neurosurgery and Psychiatry, vol. 53, pp. 976-981, 1990.

[6] C. N. Riviere, S. G. Reich, and N. V. Thakor, "Adaptive fourier modeling for quantification of tremor," Journal of Neuroscience Methods, vol. 74, pp. 77-87, 1997.

[7] N. E. Huang, Z. Shen, S. R. Long, M. C. Wu, H. H. Shih, Q. Zheng, N. Yen, C. C. Tung, and H. H. Liu, "The empirical mode decomposition and the hilbert spectrum for nonlinear and non-stationary time series analysis," Proceedings of the Royal Society of London A, vol. 454, pp. 903-995, 1998.

[8] Z. Wu and N. E. Huang, "Ensemble empirical mode decomposition: A noise assisted data analysis method," Advances in Adaptive Data Analysis, vol. 1, no. 1, pp. 1-41, 2009.

[9] K. A. Mann, F. W. Werner, and A. K. Palmer, "Frequency spectrum analysis of wrist motion for activities of daily living," Journal of Orthopedic Research, vol. 7, no. 2, pp. 304-306, 1989.

[10] J. G. Gonzalez, E. A. Heredia, T. Rahman, K. E. Barner, and G. R. Arce, "Optimal digital filtering for tremor suppression," IEEE Trans Biomed Eng, vol. 47, no. 5, pp. 664-673, 2000.

[11] J. A. Gallego, E. Rocon, J. O. Roa, J. C. Moreno, and J. L. Pons, "Realtime estimation of pathological tremor parameters from gyroscope data," Sensors, vol. 10, no. 3, pp. 2129-2149, 2010.

[12] H. J. Luinge and P. H. Veltink, "Measuring orientation of human body segments using miniature gyroscopes and accelerometers." Med Biol Eng Comput, vol. 43, no. 2, pp. 273-282, Mar 2005.

[13] J. Raethjen, M. Lindemann, H. Schmaljohann, R. Wenzelburger, G. Pfister, and G. Deuschl, "Multiple oscillators are causing parkinsonian and essential tremor." Mov Disord, vol. 15, no. 1, pp. 84-94, 2000 . 